\title{
Sandrine à Marseille : dialogue avec une transsexuelle
}

Sandrine in Marseille: dialogue with a transsexual

Jérôme Latrobe

\section{OpenEdition}

1 Journals

Édition électronique

URL : http://journals.openedition.org/clio/9675

DOI : $10.4000 /$ clio.9675

ISSN : 1777-5299

Éditeur

Belin

Édition imprimée

Date de publication : 1 mai 2010

Pagination : 197-206

ISSN : 1252-7017

\section{Référence électronique}

Jérôme Latrobe, «Sandrine à Marseille : dialogue avec une transsexuelle », Clio. Femmes, Genre, Histoire [En ligne], 31 | 2010, mis en ligne le 31 mai 2010, consulté le 19 avril 2019. URL : http:// journals.openedition.org/clio/9675; DOI : 10.4000/clio.9675

Tous droits réservés 


\section{Sandrine à Marseille : dialogue avec une transsexuelle}

Jérôme LATROBE

\section{Présentation}

Le témoignage ici reproduit est le prolongement d'une recherche exploratoire sur les enjeux identitaires liés à la corporéité. Cette recherche, effectuée en 2008 dans le cadre d'un Master universitaire s'est appuyée sur des entretiens réalisés auprès d'un échantillon de personnes transsexuelles male to female. L'une d'elle, Sandrine, a accepté de répondre à de nouvelles questions pour la revue Clio, délivrant un témoignage sur l'impact de son changement de sexe sur sa sexualité, ce qu'elle justifie par le fait « qu'elle n'a rien à cacher».

Ce document, par sa dimension singulière, n'a pas de valeur épistémologique générale et n'a pas vocation à témoigner de la sexualité de l'ensemble des personnes transsexuelles male to female. Il permet, néanmoins, d'interroger les "allants de soi", de revisiter les catégories qui servent à définir les identités et les comportements sexuels, catégories souvent encore considérées par le sens commun comme naturelles. "Construction sociale, la sexualité humaine implique nécessairement la coordination d'une activité mentale et d'une activité corporelle, qui doivent toutes deux être culturellement apprises $»^{1}$, écrit le sociologue Michel Bozon. L'activité mentale repose sur la notion de genre ou de rapports sociaux de sexe marqués par une catégorie de dominants, les hommes, et une catégorie de dominés, les femmes. Lorsque ce rapport de domination trouve ses fondements dans l'activité corporelle et est liée à l'anatomie et à la

1 Bozon $2005: 6$. 
morphologie des corps où sont distingués les mâles (le sexe fort) et les femelles (le sexe faible), ce rapport de domination entre les sexes est naturalisé.

Le témoignage de Sandrine permet d'illustrer la diversité des identités de sexe et de genre et de reconsidérer leur articulation aux catégories légales divisant l'humanité en deux ensembles distincts, les hommes et les femmes. Cette articulation est souvent simplifiée à l'extrême. Cet exemple donne à voir que, dans le vécu des individus, les contours de ces catégories sont plus confuses qu'elles ne le paraissent. Ces catégories sont acceptées et considérées comme fixes et institutionnalisées d'un point de vue légal lors de l'établissement de l'état civil. Cette classification donne lieu à des raisonnements et des schèmes binaires tendant à opposer de façon radicale les éléments de ces catégories notamment à travers les rapports de domination constitutifs de l'équilibre social. De plus, si la sexualité peut être considérée comme une activité sociale relevant de la sphère privée, elle n'en est pas moins un objet de gouvernement par les pouvoirs publics. Ainsi, l'hétérosexualité trouve ses fondements dans la norme conjugale et ses fonctions reproductives. Comme le souligne Daniel Borillo, « en quelque sorte il n'est pas seulement question de fabriquer des corps mais aussi de subjectiver des consciences et d'encourager un certain type de représentation de l'activité sexuelle $»^{2}$.

Sandrine, née avec un appareil génital masculin et déclarée mâle à la naissance, a aujourd'hui 42 ans. Elle s'est fait opérer à 37 ans pour mettre en accord son ressenti de femme avec les attentes sociales. Avant ce changement de sexe, Sandrine a grandi en tant que garçon, un garçon qu'elle qualifie d'androgyne. Bien que son apparence physique ait contribué à entretenir une certaine ambigüité sur son identité de genre, sa constitution anatomique d'homme ainsi que son attirance physique pour les hommes ont amené Sandrine à découvrir la sexualité à travers des rapports homosexuels. Il est à noter que Sandrine n'a jamais eu recours à la prostitution, ni en tant que client ni en tant que personne prostituée pour financer son changement de sexe. Lorsqu'elle a décidé de s'engager dans le processus de ce

2 Borillo 2004 : 191. 
changement, Sandrine a choisi de rompre la relation amoureuse dans laquelle elle était engagée, afin de mener ce « parcours » en évitant les éventuels commentaires de son compagnon de l'époque.

\section{Entretien}

JL

Ta sexualité et/ou ton rapport à la sexualité ont-ils été transformés en même temps que ta transformation corporelle?

\section{Sandrine}

Après l'opération, l'intérêt, c'est l'union physique, le fait qu'il y ait pénétration. J'ai l'impression de faire l'amour vraiment, ce que je ne faisais pas avant. L'opération a été nécessaire. En fait non parce que je ne m'en rendais pas compte. Je n'en avais eu qu'un aperçu en tant qu'homme dans des relations homosexuelles anales qui me déplaisaient. Je faisais ma vie comme ça.

Par exemple, je ne pensais pas que les seins étaient un élément érotique et de séduction très important. Quand j'ai eu mes seins quand je suis sortie de la clinique je me suis fait brancher au moins 34 fois, donc à ce moment-là j’ai compris que c'était important. Avant je séduisais quand même des hommes mais pas à ce rythme là. Là c'était vraiment important. Ca a changé quelque chose d'important. Je ne savais pas trop ce que l'opération allait m'apporter sexuellement et l'union, la pénétration, m'a apporté un plus, qui m'est apparu très important. Lors du rapport, je suis plus passive (si je veux !), et eux plus actifs. C'est plus agréable pour moi. Moi-même, à part la pratique où j'aime bien être dessus (qui est plus active !), à part ça je reste plus passive. Avant l'opération j'étais dessous et maintenant je suis dessus. Dessus, j'atteins mieux l'orgasme.

JL

La personne qui est dessus est-elle plutôt en position de domination pour toi ? 


\section{Sandrine}

Oui. Je pense. En fait j’ai basculé. Je suis passé d'une position de dominé en étant garçon car j’aimais encore moins pénétrer qu'être pénétré, à une position de domination en étant femme. Est-ce le fait d'être androgyne qui me pousse à rééquilibrer quelque chose ?

JL

...afin de retrouver un équilibre lié à la discordance entre le choix de tes positions dans les rapports sexuels avant et après opération et la position «conventionnelle » déterminée par ton sexe anatomique (avant et après opération)?

\section{Sandrine}

Oui c'est ça, j'étais garçon et je me positionne comme une fille, passivement, et maintenant je suis femme et je positionne comme un garçon, plutôt actif. Ça me permet peut-être de retrouver quelque chose d'androgyne, quelque chose des deux sexes en même temps.

JL

Les seins ou les pratiques buccales sont des pratiques ou des zones érogènes nouvelles ou que tu as développées?

\section{Sandrine}

Les seins c'était bien, donc ça m'a apporté autre chose au niveau de la séduction et de l'excitation de mes partenaires et au niveau buccal, c'était une des rares pratiques avec les caresses que je faisais lors de mes rapports homo. Et je me demande si ce n'est pas une des pratiques qui fait qu'il y a des hommes qui s'accrochent aujourd'hui. Il faut avoir eu un sexe masculin pour comprendre ce qu'est une fellation. Donc ça c'est un atout supplémentaire que j'ai par rapport aux femmes biologiques.

JL

Est-ce que cette nouvelle sexualité, puisque tu disais que c'était différent, te confirme dans ton identité de femme ou est-ce que tu sépares ta sexualité de ton identité de femme? 


\section{Sandrine}

Oh non, c'est pas que ça me confirme mais c'est un tout, enfin ça confirme que j'ai bien fait de me faire opérer. Quand je l'ai fait j'avais une apparence féminine et mon rapport à mon sexe anatomique était quelque chose de très intime.

Donc j'existais en tant que femme socialement sans avoir un sexe féminin donc ça prouve que c'est pas vraiment déterminé naturellement. Le fait d'avoir le sexe féminin fait qu'il n'y a même plus d'ambiguïté dans ma tête. Pour moi le mot transsexuel ne me convient pas bien. Je ne me sens pas transsexuelle. Mais bon je l'assume. Il est évident que telle que je suis, je l'ai toujours été. Le changement de sexe ne m'a pas changée fondamentalement. C'est une suite logique mais j'aurais peut-être aussi pu finir ma vie comme ça sans opération. Ça m'a amené un confort, c'est vrai par rapport au milieu social. Qui est très important mais aussi ça me convenait. Mais l'androgyne que j'étais à 20 ans qui plaisait à tout bout de champ, quand t'arrives à 50 ans, ça n'a plus le même impact sexuellement attractif que quand tu as 20 ans. J'imagine que dans peu de temps ça se serait étiolé. J'aurais dépéri ou j'aurais déprimé. Je pense qu'il était nécessaire que je change de sexe pour finir ma vie ou pour avancer dans ma vie.

Je l'ai fait seule, après ma rupture d'avec mon copain d'alors. Je voulais être seule avec mes ressentis vis à vis des autres pour pouvoir bien m'évaluer sans être influencée dans mes choix par quelqu'un d'autre. Je n'imaginais pas être avec quelqu'un à qui j'ai plu en garçon et continuer à lui plaire en femme. J'aurais eu un doute permanent sur son regard vis-à-vis de moi.

JL

Y a-t-il eu des changements dans ton rapport de séduction des hommes?

\section{Sandrine}

Moi de toute façon je suis plutôt cash, tu vois. Je ne l'étais pas avant. Mais maintenant oui. Je ne veux pas louvoyer. Je suis assez directe même dans mes rapports avec les hommes. Souvent ils sont déstabilisés. Ils prennent des précautions et moi je leur dit : «bon tu 
es venu pour faire l'amour?» Eux ils imaginent qu'ils vont devoir louvoyer pour finir dans un lit mais moi je passe très vite à l'essentiel.

JL

Tu penses que c'est le fait que tu as été garçon avant ou... ?

\section{Sandrine}

Oui certainement. Je pense que c'est une question d'éducation. Vu que les femmes ne peuvent pas se donner comme ça. Elles sont obligées par tout un tas de protocoles : dire oui et penser non et vice versa. Quand j'étais garçon les rapports s'embrayaient tout de suite quoi. Ou ça passe ou ça ne passe pas. Ça passe très très vite. Quand j'étais à Paris, les rencontres, ça allait très très vite et puis le nombre de garçons que tu rencontres. Les hommes ont une timidité vis-à-vis des femmes qui est assez forte et une timidité vis-à-vis de l'homosexualité qui est assez forte. Il y a toujours eu de la retenue de leur part. Ils n'ont jamais été en position de force. Le fait que j’étais androgyne me donnait un pouvoir sur eux. Ils ne connaissaient pas les codes. Ils étaient habitués aux codes des femmes mais ils ne connaissaient pas les codes spéciaux.

JL

Quand tu es relativement directive lors de l'étape de séduction, il s'agit de faciliter les choses à ton partenaire en lui donnant les codes pour gagner du temps, c'est pour lui faire plaisir à lui ou c'est aussi pour te faire plaisir à toi ?

\section{Sandrine}

C'est aussi pour me faire plaisir, bien sûr. S'il faut passer à l'essentiel on y va! Je pourrais avoir des tactiques en me disant « oui il me plait bien, je le reverrai demain ». Mais non. Et puis je ne suis pas en démarche affective. Je n'ai pas besoin de m'accrocher à un homme. J'étais très choqué quand j'étais garçon, j'étais très étonné dans mon rapport avec les hommes, parce que pour moi un homme c'est quelqu'un sans lendemain mais moi je suis tombée sur des hommes qui voulaient tous des lendemains. Je n'essaie pas justement 
de créer une dynamique de lendemain. S’il y en a il y en a. Je ne cherche pas à créer ce type de relation.

D'habitude, les hommes ils disent: " oui tu sais avec moi il ne faut pas s'accrocher». Alors je leur dis : "tu sais avec moi il ne faut s'accrocher non plus ». Ils sont étonnés. Ils ont peut-être rencontré des femmes qui pleurnichaient, mais moi je ne suis pas du tout en demande affective. J'aime et je suis aimée. C'est ça. J'ai été très aimé. Que ce soit dans la famille, par mon grand-père, par tout le monde. Je n’ai pas été rejeté du tout.

\section{Commentaire}

Sandrine s'est toujours définie comme androgyne. Bien que née avec un sexe anatomique de mâle, elle a toujours considéré son apparence physique comme ambiguë, indéterminée entre le féminin et le masculin. Il est à noter que cette ambigüité a été entretenue par ses parents qui l'ont laissée se vêtir et se comporter d'une manière relativement asexuée.

La détermination sociale de sexe et de genre liée à son sexe anatomique de naissance ne semble pas avoir été opérante lors de la socialisation et de la construction de l'identité de genre de Sandrine. En effet, cette détermination masculine légale due à son sexe anatomique d'origine ne lui a pas permis de se construire socialement dans l'indétermination de genre qu'elle aurait souhaitée. Si Sandrine a choisi de transformer son corps en se soumettant à une expertise psychiatrique qui lui a donné accès aux traitements hormonaux et chirurgicaux nécessaires au changement de sexe, cela a été dans un souci d'intégrer pleinement une identité sociale de femme aux dépens d'une identité sociale « androgyne » non reconnue légalement.

Sa posture construite sur son androgynie, a été tolérée par son environnement social et familial. À trente ans, pour Sandrine, la perspective de vieillir dans ces conditions ne lui sont pas apparues comme possibles et a donné une dimension nouvelle à sa sexualité. Bien que se sentant et se vivant aussi bien garçon que fille, Sandrine a été amenée à l'âge adulte à choisir un des deux sexes afin de tenir une 
position sociale. Elle a aussi cherché à mettre en cohérence son sexe anatomique et son identité sociale afin d'éviter toute « surprise » à ses partenaires. C'est au prix de ce choix que Sandrine a pu tenir une place socialement acceptée dans ses relations sociales et ses rapports sociaux de sexe ainsi que dans ses rapports sexuels, en jouant de manière contradictoire avec les pôles de la passivité et de l'activité. D'une part, nous pouvons considérer que la sexualité est passée d'un statut de jeu dans les rapports de sexe à un statut d'épanouissement sexuel devant correspondre à des normes structurantes. D'autre part, cet objectif d'épanouissement sexuel a imposé à Sandrine un effort de discipline et de conformisme pour se faire reconnaitre en tant que femme. Cet effort est en tension, apparemment paradoxale, avec une tendance à l'originalité liée à son sexe de naissance et à son parcours spécifique. L'expression de cette originalité peut invalider les efforts de conformisme effectués pour " passer $^{3}$ » socialement en tant que femme.

Après avoir vécu un apprentissage de la sexualité à travers des rapports homosexuels, Sandrine accepte de nous livrer les transformations de sa sexualité suite à son opération qui l'a fait passer d'«homme» à «femme». Elle présente d'une part les apports de l'intervention chirurgicale en termes sensoriels et relationnels notamment à travers la découverte de la pénétration vaginale comme source de plaisir. D'autre part, elle commente la place qu'elle occupe désormais dans ses rapports avec ses partenaires masculins. Néanmoins, nous pouvons comprendre dans le discours de Sandrine que, si elle est aujourd'hui une femme et que ce nouveau corps lui a procuré des apports positifs qu'elle ne conteste pas dans le domaine de ses rapports de séduction et de ses rapports sexuels, elle continue de penser que le fait d'avoir été garçon peut constituer un atout pour certaines pratiques. Le fait d'acquérir et de s'approprier l'ensemble des codes de la sexualité féminine ne constitue pas, pour elle, un objectif. Sandrine semble jongler avec les représentations qu'elle a des sexualités masculine et féminine en tentant de les mettre simultanément en tension. C'est à travers ces représentations mises en scène dans le cadre de la sexualité que Sandrine s'autorise à se penser

3 Garfinckel 2007 : 205. 
comme fondamentalement des deux sexes en se qualifiant d'androgyne.

Bien qu'ayant fait le choix d'appartenir à une seule catégorie sociale, Sandrine marque une certaine distance avec la catégorie de femmes en soulignant la distinction qui existe entre elle-même et les «femmes biologiques». L'opération de changement de sexe lui a apporté la légitimité sociale d'être une femme, cela dit la question de son identité de genre intime reste invariablement ambigüe. Sandrine ne s'identifie pas intimement à la catégorie des transsexuels dans le sens où cette catégorie signifie, pour elle, passer d'un sexe à l'autre. Sandrine s'identifie, aujourd'hui à une femme hétérosexuelle. Il convient de préciser ici que depuis son changement de sexe, elle n'a jamais eu de rapports sexuels avec une autre femme ni avec une autre personne transsexuelle. Malgré cela, son identité de genre intime ne semble pas bouleversée, y compris dans l'activité sociale qu'est la sexualité dans laquelle la détermination due au sexe anatomique implique de la part des partenaires des comportements socialement intégrés et attendus. Les travaux de Michel Foucault sur les questions de pouvoir et de gouvernement nous incitent à souligner la subjectivité qui est à l'œuvre quand les acteurs définissent eux-mêmes leur(s) identité(s) et délivrent une parole sur ce « gouvernement de soi par soi dans son articulation avec les rapports à autrui ${ }^{4} »$ qu'est la sexualité. L'étude de la sexualité, lieu d'articulation privilégié de l'identité sociale (par le corps), de l'identité de genre intime et des normes sociales, met en évidence, d'une part, toute la complexité de la notion de rapports de sexe qui ne peut se résoudre que grossièrement dans les interactions entre les deux catégories légales universelles. D'autre part, elle amène à souligner la valeur heuristique de la notion de corporéité et à en mesurer les enjeux sociaux et individuels.

$4 \quad$ Foucault $1994: 214$. 


\section{Bibliographie}

BORILLO Daniel, 2004, «La surveillance juridique des pratiques sexuelles légitimes. L'institution de la norme conjugale ", in Didier FASSIN \& Dominique MEMMI (dir.), Le gowvernement des corps, Paris, Éditions de l'École des Hautes Etudes en Sciences Sociales.

BOURDIEU Pierre, 1998, La domination masculine, Paris, Seuil.

BOZON Michel, 2005, Sociologie de la sexualité, Paris, Armand Colin.

FOUCAULT Michel, 1994, Dits et écrits, résumé du cours an Collège de France de 1980, t. 4, Paris, Gallimard.

GARFINKEL Harold, 2007 [1967], Recherches en ethnométhodologie, Paris, PUF. 